\title{
Uma metodologia para constituir redes de apoio aos universitários em risco de evasão durante a pandemia da Covid-19
}

A methodology to build support networks for university students at risk of dropping out during the

\author{
Covid-19 pandemic
}

Una metodología para construir redes de apoyo para estudiantes universitarios en riesgo de deserción durante la pandemia de Covid-19

Letícia Martins Raposo

ORCID: https://orcid.org/0000-0003-0613-5582 Universidade Federal do Estado do Rio de Janeiro, Brasil E-mail: leticia.raposo@ uniriotec.br

Maria Beatriz Assunção Mendes da Cunha ORCID: https://orcid.org/0000-0002-9669-4232 Universidade Federal do Estado do Rio de Janeiro, Brasil E-mail: beatriz.cunha@uniriotec.br r

Maria Tereza Serrano Barbosa ORCID: https://orcid.org/0000-0003-0977-5921 Universidade Federal do Estado do Rio de Janeiro, Brasil E-mail: tserranobarbosa@gmail.com

Gladson Octaviano Antunes

ORCID: https://orcid.org/0000-0003-3692-374X Universidade Federal do Estado do Rio de Janeiro, Brasil E-mail: gladson.antunes@uniriotec.br

Morganna Carmem Diniz

ORCID: https://orcid.org/0000-0002-3238-5289

Universidade Federal do Estado do Rio de Janeiro, Brasil

E-mail: morganna@ uniriotec.br

Heloisa Helena Albuquerque Borges Quaresma Gonçalves

ORCID: https://orcid.org/0000-0002-8025-2288 Universidade Federal do Estado do Rio de Janeiro, Brasil E-mail: heloborges@uniriotec.br

Felipe Rafael Ribeiro Melo ORCID: https://orcid.org/0000-0002-1482-8533 Universidade Federal do Estado do Rio de Janeiro, Brasil E-mail: felipe.ribeiro@uniriotec.br

Michel Cambrainha de Paula ORCID: https://orcid.org/0000-0002-3552-2488 Universidade Federal do Estado do Rio de Janeiro, Brasil

E-mail: michel.cambrainha@uniriotec.br

Diana Cristina Alves da Penha Grijó dos Santos

ORCID: https://orcid.org/0000-0002-7336-3959 Universidade Federal do Estado do Rio de Janeiro, Brasil E-mail: diana.penha@unirio.br Jefferson Elbert Simões

ORCID: https://orcid.org/0000-0002-0359-118X Universidade Federal do Estado do Rio de Janeiro, Brasil E-mail: jefferson.simoes@uniriotec.br

\begin{abstract}
Resumo
Os objetivos deste trabalho consistiram em desenvolver e aplicar uma metodologia a fim de identificar estudantes universitários com maior risco de evasão durante a pandemia de covid-19 e propor ações minimizando este risco. A proposta apresentada utilizou a amostragem por Bola de Neve e as redes de contato para entender como atuar para aumentar a motivação acadêmica. Uma metodologia de pesquisa-ação foi aplicada à comunidade de estudantes de ciências exatas e tecnologia do CCET/UNIRIO e, com os dados coletados, identificou-se os alunos que poderiam estar em maior risco de evasão. Verificou-se que $50 \%$ dos estudantes respondentes apontaram que as alterações na vida pessoal durante a pandemia da covid-19 poderiam impedir a continuação no curso, sendo este o principal risco de evasão identificado. Ainda, $41 \%$ dos respondentes indicaram a falta de motivação acadêmica como razão para interromper o curso. A partir destas e de outras informações, foi possível avaliar o quanto estes estudantes estavam
\end{abstract}


conectados e engajados com a continuidade da sua vida acadêmica e identificar ações que poderiam contribuir para manter o seu vínculo com a universidade. Por fim, foram realizados eventos de acolhimento e diálogo com participação de docentes, técnicos e discentes, visando diminuir as chances de evasão dos alunos identificados. Estes eventos sinalizaram a expectativa dos discentes por mais diálogo em sala de aula, o que subsidiou a continuidade do projeto.

Palavras-chave: Covid-19; Evasão universitária; Bola de neve; Redes de apoio; Pesquisa-ação.

\begin{abstract}
The goals of this study consisted of the development and application of a methodology aimed at identifying college students with a higher risk of dropping out during the covid -19 pandemic and the proposal of actions reducing this risk. We used snowball sampling and contact networks to understand how to act to enhance academic motivation. A quantitative action-research methodology was applied to the community of students from STEM courses at CCET/UNIRIO and, with the data collected, we identified students that could have a higher dropout risk. We verified that $50 \%$ of responding students pointed out that changes in their personal lives caused by the covid -19 pandemic could prevent the continuity of their studies, this being the main dropout risk identified. Also, $41 \%$ of responding students identified a lack of academic motivation as a reason for dropout. From this and additional information, we were able to evaluate how connected and engaged with their academic lives these students were and identify actions that could contribute to maintaining their bond with the university. Finally, we held welcoming and dialogue events featuring teachers, staff, and students, in an attempt to reduce the dropout risk of the identified students. These events highlighted the students' expectations for more dialogue in the classroom, which supported the follow-up of the project.
\end{abstract}

Keywords: Covid-19; University dropout; Snowball sampling; Support networks; Action-research.

\title{
Resumen
}

Los objetivos de este trabajo fueron desarrollar y aplicar una metodología con miras a identificar a los estudiantes universitarios con mayor riesgo de deserción durante la pandemia de covid-19 y proponer acciones para minimizar este riesgo. La propuesta presentada utilizó el muestreo de bola de nieve y las redes de contacto para comprender cómo actuar para aumentar la motivación académica. Se aplicó una metodología de investigación-acción a la comunidad de estudiantes de ciencias exactas y tecnología del CCET/UNIRIO y, con los datos recolectados, se identificaron los estudiantes que podrían estar en mayor riesgo de deserción. Se encontró que el $50 \%$ de los estudiantes encuestados señalaron que los cambios en la vida personal durante la pandemia de covid-19 podrían impedir la continuación en el curso, que es el principal riesgo de deserción identificado. Aún así, el $41 \%$ de los encuestados señaló la falta de motivación académica como motivo para interrumpir el curso. A partir de esta y otras informaciones, fue posible evaluar cuánto estos estudiantes estaban conectados y comprometidos con la continuidad de su vida académica e identificar acciones que pudieran contribuir a mantener su vínculo con la universidad. Finalmente, se realizaron eventos de bienvenida y diálogo con la participación de profesores, técnicos y estudiantes, con el objetivo de reducir las posibilidades de evasión de los estudiantes identificados. Estos eventos señalaron la expectativa de los estudiantes por más diálogo en el aula, lo que apoyó la continuidad del proyecto.

Palabras clave: Covid-19; Deserción universitaria; Bola de nieve; Redes de apoyo; Investigación-acción.

\section{Introdução}

A pandemia da covid-19 já é considerada a maior crise humanitária dos últimos cem anos e teve consequências econômicas de grande impacto para o mundo. Assim como em outras epidemias, já era esperado, desde o início, que ela atingiria de forma mais grave a população mais pobre (Ahmed et al., 2020). No Brasil, a pandemia chegou em um momento em que o país atravessava uma grave e prolongada crise econômica, atingindo de forma desigual a base da pirâmide social que, além de ter perda de renda e trabalho, estava mais sujeita à contaminação e ao desenvolvimento de casos graves (Carvalho, 2020). Um estudo recente demonstrou que os indicadores de letalidade por covid-19, no município do Rio de Janeiro, são maiores nos bairros com menor desenvolvimento social (Miranda et al., 2020).

Nas universidades públicas, a substituição dos vestibulares pelo Exame Nacional do Ensino Médio (ENEM), a criação do Sistema de Ingresso Superior Unificado (SiSU), a lei de cotas (lei no 12.711, de 29 de agosto de 2012), o maior número de vagas nos cursos noturnos e o aumento das políticas de assistência estudantil ampliaram a proporção de estudantes oriundos das classes menos favorecidas, majoritariamente negros (pretos e pardos) (Godoi \& Santos, 2021). Esta democratização mudou a cor e a composição social da sala de aula e trouxe impactos positivos ao permitir a mobilidade social e favorecer o encontro entre quem não teria a oportunidade de se conhecer e conviver em igualdade de condições. Por outro lado, os mesmos 
estudantes que se beneficiaram desta ampliação são os que mais apresentam dificuldades quando tentam conciliar os estudos com o trabalho, seja pela grande quantidade de horas que precisam dedicar ao trabalho, seja pela ausência de uma rede de apoio social e/ou familiar. A falta de estrutura da própria Universidade, que via de regra privilegia os estudantes com dedicação integral e apoio familiar, empurra naturalmente os estudantes mais pobres para fora. Se somarmos a isso os efeitos diretos e indiretos da pandemia da covid-19, este índice de evasão tende a aumentar. Em recente artigo, Mussliner et al. (2021) ressaltam os múltiplos impactos da evasão universitária por representar uma frustração não só para o discente, mas também para sua família, ao desfazer sonhos, tal como para os docentes, ao se sentirem frustrados no papel de educador, e ainda para a sociedade, ao não contar com os profissionais qualificados.

Desde as primeiras medidas de distanciamento social até o atual momento, em que a maior parte das atividades ainda está sendo realizada de maneira remota, foi e ainda é fundamental que a Universidade tenha um olhar diferenciado para esses discentes jovens e adultos que, de uma hora para a outra, além de estarem, como todos, distanciados da vida acadêmica, vivenciam o dia a dia dessa realidade trágica, que é mais intensa nos seus locais de moradias e entre seus familiares e vizinhos. $\mathrm{O}$ risco de que a dura realidade econômica e sanitária interrompa de forma mais permanente os seus vínculos com a universidade deve ser considerado de forma prioritária, exigindo a formulação e execução de ações concretas.

As primeiras ações que ocorreram na Universidade Federal do Estado do Rio de Janeiro (UNIRIO) envolveram o envio de questionários online que tentavam identificar as condições de acesso à Internet dos estudantes com vistas a viabilizar a implantação de aulas remotas. Contudo, essa estratégia se revelou paradoxal, pois os estudantes deveriam ter acesso à Internet para poder informar aos seus cursos que não possuíam acesso à Internet. Nesse contexto, um grupo de docentes e técnicos do Centro de Ciências Exatas e Tecnologia (CCET), preocupado com estes discentes em maior vulnerabilidade socioeconômica e que pudessem estar invisíveis e inalcançáveis pelos primeiros questionários eletrônicos, iniciou discussões sobre uma proposta de metodologia para alcançar tais estudantes no próprio centro, com possibilidade futura de expansão para outras unidades acadêmicas da UNIRIO.

Em meados de agosto de 2020, este grupo submeteu para apreciação do Comitê de Ética em Pesquisa (CEP UNIRIO) o projeto intitulado "Acessando os discentes em risco de evasão após a covid-19 e constituindo redes de apoio", e aprovado sob o número 37010620.6.0000.5285. Seu objetivo geral era minimizar o risco de evasão dos estudantes mais atingidos social e economicamente pela pandemia, a partir da sua identificação e engajamento em redes de apoio. Na concepção e formulação do projeto, uma questão que se colocava era o quanto era possível minimizar o risco de evasão deste grupo discente a partir de espaços de trocas, vivências universitárias, seminários, eventos e reuniões. Ou seja, considerou-se que os vínculos sociais com integrantes da comunidade universitária poderiam contribuir, em certa medida, para a permanência desses estudantes na universidade.

Diante deste contexto, este artigo apresenta uma metodologia desenvolvida para localizar os estudantes do CCET/UNIRIO em maior risco de evasão e identificar suas redes de apoio. Por meio dela, avaliamos o quanto estes estudantes estão conectados e engajados com a continuidade da sua vida acadêmica e identificamos ações que poderiam contribuir para manter o seu vínculo.

\section{Metodologia}

A proposta que apresentamos neste artigo consiste em explorar a rede social universitária utilizando amostragem por Bola de Neve (Goodman, 1961). Para isso, os dados foram coletados para identificar alunos em situação de vulnerabilidade, para construir as redes de contato pertinentes, e planejar ações concretas visando diminuir as chances de evasão dos alunos identificados. As etapas propostas foram adaptadas do estudo desenvolvido por Hindhede e Aagaard-Hansen (2017), que utilizaram métodos de amostragem por Bola de Neve e análise de redes de contato (redes sociais) a fim de construir redes que 
permitissem avaliar o nível de participação de indivíduos e grupos em uma comunidade pertencente a um bairro vulnerável de uma cidade na Dinamarca. A seguir, são descritas as quatro etapas realizadas neste trabalho.

\section{Etapa 1: Alinhamento e uniformização da entrevista sensível a ser feita com cada estudante}

Etapas de preparação de uma pesquisa que envolve entrevistas e questionários são vitais. A qualidade das informações coletadas podem variar tanto pelo modo como a entrevista é realizada - por correio, por telefone ou pela Internet - quanto pela forma de condução da entrevista pelo entrevistador. Para estabelecer um padrão na forma como as respostas são registradas, é importante que a etapa de treinamento e uniformização das entrevistas seja cautelosa.

\section{Etapa 2: Recrutamento por amostragem por Bola de Neve}

Esta etapa tem como objetivo recrutar os possíveis discentes em risco de romper seus vínculos com a vida acadêmica. Para isto, é necessário constituir uma amostra inicial de estudantes potencialmente em condição de vulnerabilidade, denominada semente (Figura 1).

Figura 1: Discentes socioeconomicamente vulneráveis selecionados para compor a primeira amostra da estratégia de amostragem por Bola de Neve.

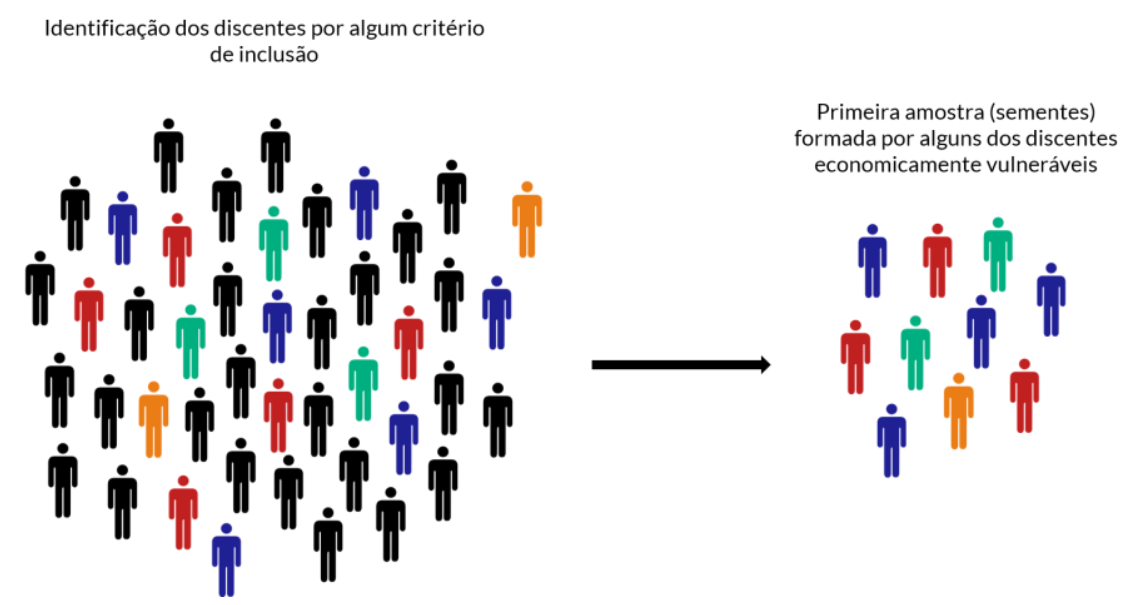

Fonte: Autores.

A identificação desses estudantes pode ser realizada tanto por meio de dados fornecidos pela própria universidade, quanto do conhecimento tácito adquirido pelos servidores atuantes nos cursos em questão. Após este passo, cada discente indicado pertencente à amostra semente deve ser convidado a responder o questionário e indicar outros estudantes que eles acreditem estar em situações semelhantes, a partir de sua própria rede pessoal, e assim sucessivamente (Figura 2) - esta é, efetivamente, a amostragem por Bola de Neve da rede social universitária. Esta estratégia de amostragem foi selecionada pois, segundo Bernard (2005), ela constitui um método útil para se estudar populações difíceis de serem acessadas. Além disso, ela tem sido considerada econômica, relativamente rápida, eficiente e eficaz em vários sentidos, sendo muito utilizada para preencher lacunas em relação ao conhecimento de situações sociais mais obscuras (Atkinson \& Flint, 2001). Este tipo de amostragem pode ser utilizado para a disseminação de informações e estabelecimento de uma rede de apoio visando auxiliar no bem-estar de populações escondidas ou difíceis de encontrar. No entanto, por depender das escolhas subjetivas dos indicados, a amostragem por Bola de Neve pode excluir indivíduos não conectados com nenhum dos recrutados (Griffiths et al., 1993). 
Figura 2: Primeira onda da amostragem por Bola de Neve. As sementes (indivíduos maiores) indicam novos nomes (indivíduos menores), independentes se estes recebem auxílio da instituição.

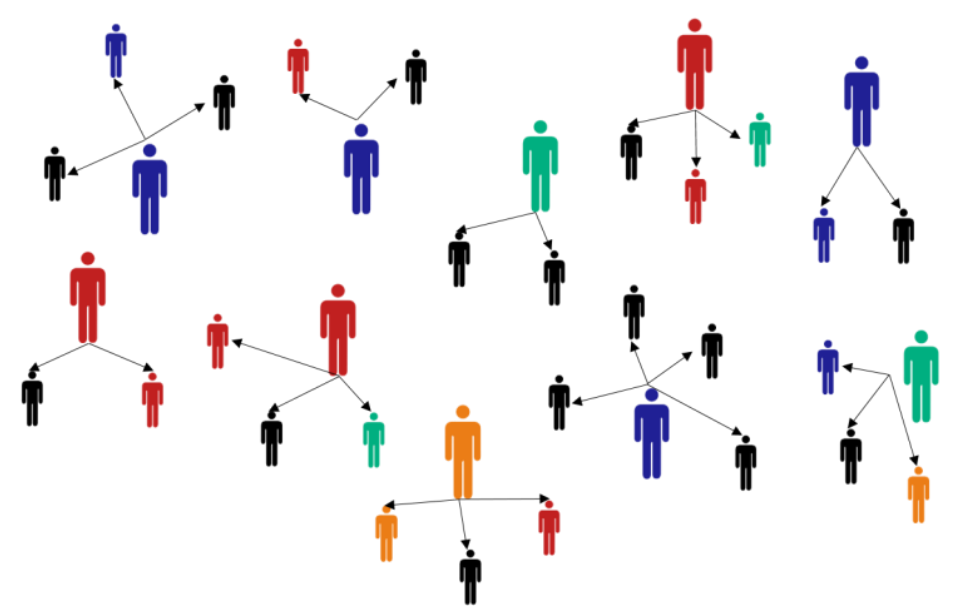

Fonte: Autores.

A dinâmica descrita acima deve ocorrer até o quadro de amostragem se tornar saturado, não havendo novos nomes a serem indicados, ou até que os nomes encontrados não tragam informações novas ao quadro de análise.

\section{Etapa 3: Constituição das redes de contatos}

A cada contato realizado na etapa anterior, o discente indicado deve ser convidado a participar do projeto, e os que desejarem participar devem preencher um questionário, a fim de levantar dados sobre suas condições socioeconômicas. Estes dados serão utilizados mais adiante para identificar o perfil de vulnerabilidade e o risco de rompimento de cada discente com a vida acadêmica.

\section{Etapa 4: Construção das redes de contatos}

A metodologia de redes de contatos pode ser utilizada a partir de uma amostragem por Bola de Neve para descrever e analisar interações entre um conjunto definido de atores, baseando-se no pressuposto de que os unidos por algum interesse interagem entre si (Barbosa et al., 2000). Essa teoria considera as relações sociais sendo mais importantes para explicar fenômenos sociais do que os atributos individuais, podendo servir para avaliar a cobertura de projetos e identificar a centralidade de indivíduos em casos de novas intervenções planejadas Hindhede e Aagaard-Hansen (2017).

Por meio do questionário, fornecido na etapa anterior, os discentes também devem fornecer nomes de discentes e servidores com os quais eles possuem maior proximidade pessoal (Figura 3). Estas pessoas são de extrema importância para a manutenção dos discentes na universidade, pois representam pontos de apoio social para os mesmos. Nesta etapa, devem ser levantados os contatos fornecidos e os relacionamentos entre os discentes e seus contatos, representando indicações de vulnerabilidade ou contatos de afinidade. Neste momento, é possível fazer uma análise desta rede, levantando grupos de pessoas (discentes e servidores) que constituem subcomunidades próprias, pessoas em posição de centralidade na rede social, etc. É importante que, durante esta análise, o anonimato dos membros desta rede seja preservado.

Os dados da rede geralmente são exibidos como pontos e linhas em um gráfico de rede. Os atores são representados como pontos no diagrama e são chamados de nós ou vértices. As conexões são as arestas ou os laços entre os nós. O relacionamento ou vínculo é um fluxo de recursos que pode incluir suporte social, tempo, informações e atividades compartilhadas (Scott, 1988). Estas ligações e relacionamentos tendem a ser dinâmicos, podendo se alterar com o tempo (Borgatti et al., 2018). 
Figura 3: Os discentes que indicaram desejo de participar do projeto (marcação em verde) informam novos nomes de discentes em situações de risco de rompimento da vida acadêmica e respondem um questionário de avaliação. Os discentes que recusaram participar do estudo (marcação em amarelo) continuam na amostra, mas não respondem ao questionário, sendo a indicação de novos nomes opcional.

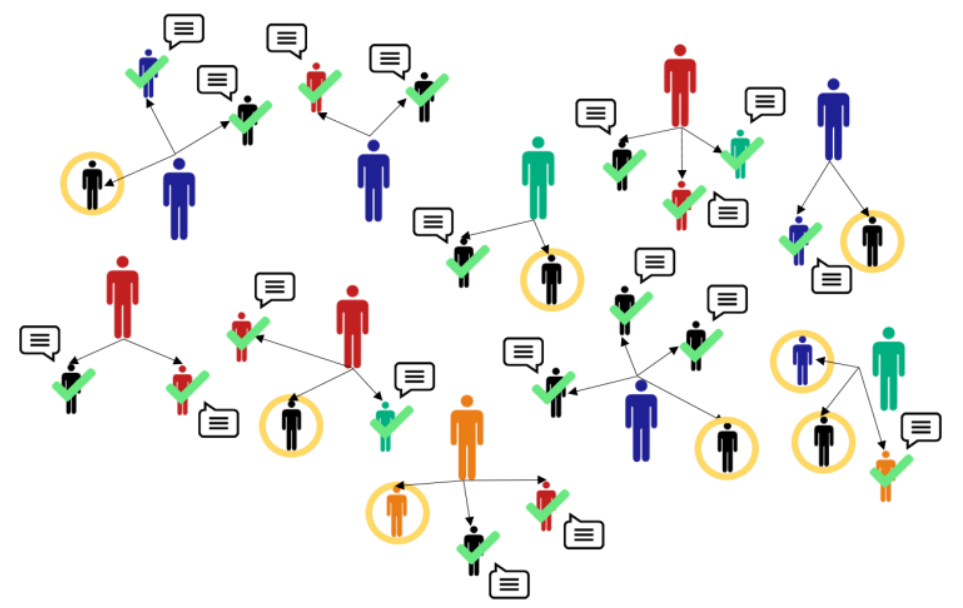

Fonte: Autores.

\subsection{Cenário de Aplicação}

Para aplicar esta metodologia no âmbito do CCET/UNIRIO, foi realizada uma pesquisa quanti-ação, que caracterizase por envolver coleta de dados com o objetivo de modificar uma realidade (Barbosa et al., 2016). Os dados foram coletados por meio da aplicação de um questionário, no mês de setembro de 2020, direcionado aos estudantes matriculados nos três cursos presenciais de graduação do CCET/UNIRIO: Bacharelado em Sistemas de Informação, Licenciatura em Matemática, e Bacharelado em Engenharia de Produção. Devido à metodologia de amostragem por Bola de Neve, não houve estimativa de tamanho da amostra.

A seguir, discutimos aspectos importantes que foram considerados ao aplicar cada etapa da metodologia proposta. Na etapa de uniformização da pesquisa, em se tratando de uma pesquisa de tema sensível sobre vulnerabilidade nos vínculos dos estudantes com a Universidade, que pode ser afetada inclusive pela falta de acesso a ferramentas de Internet, a entrevista poderia ocorrer por meio de diferentes meios de comunicação. Antes de realizar os contatos, considerou-se que conversar sobre a própria vulnerabilidade não era a mais confortável das situações. Diante disso, o primeiro contato teria o potencial de aproximá-los ou de afastá-los. Em contraponto ao distanciamento (e, por vezes, certa frieza) das comunicações escritas, a equipe decidiu pela realização de chamadas telefônicas.

No caso do discente não ter acesso a serviços de Internet ou ter dificuldade para acessar o formulário de entrevista, a mesma deveria ocorrer por meio de contato telefônico. Apesar da existência de risco mínimo de exposição dos dados dos respondentes, esta é uma pesquisa de tema sensível. Portanto, um alinhamento prévio foi realizado entre os entrevistadores, para a condução das entrevistas e apresentação do projeto aos discentes vulneráveis, direcionando o foco para as necessidades do participante. Se não fosse possível estabelecer uma entrevista naquele momento, outro contato telefônico deveria ser realizado.

As abordagens dos entrevistadores foram cuidadosamente definidas e levaram em consideração as orientações apontadas em Dempsey et al. (2016). As padronizações, alinhamentos e uniformizações ocorreram a partir de várias reuniões da equipe de pesquisa.

Durante o processo de recrutamento e entrevista, optou-se por construir a semente a partir de duas fontes: 
1. A lista dos estudantes dos cursos do CCET contemplados com bolsas ou auxílios de assistência estudantil fornecidas pela UNIRIO: auxílio alimentação (AA), auxílio moradia (AM), bolsa de incentivo acadêmico (BIA) e auxílio moradia mais bolsa de incentivo acadêmico (BIA+AA).

2. Uma lista de indicações fornecidas pelas Direções de Escola e pelo Núcleo de Apoio Pedagógico (NAPE).

Os estudantes foram contatados por meio de ligações telefônicas, feitas por duas pedagogas do NAPE. Durante a ligação, cada discente foi informado sobre os objetivos e a dinâmica do projeto, sendo convidado a participar dele. Os discentes que aceitaram participar receberam, por e-mail, o questionário, criado na plataforma Google Formulários. O questionário possui perguntas abertas e fechadas e é dividido em duas partes: a primeira parte abordou questões relativas ao aluno (curso, idade, gênero, estado civil, constituição familiar) e às condições que poderiam ser desencadeadoras do rompimento do seu vínculo com a universidade; a segunda parte englobou perguntas abertas em que o aluno indicava outros discentes em situação de vulnerabilidade e colegas, docentes e técnicos-administrativos com os quais possuía maior proximidade.

A preservação do anonimato dos sujeitos respondentes ou indicados foi garantida a partir da omissão dos respectivos nomes, uso de pseudônimos ou substituição dos nomes por letras e números. As informações obtidas foram exportadas para uma planilha Microsoft Excel 2013 (Microsoft Corporation, Redmond, Washington) e analisadas no software R (versão 4.0.4, R Foundation for Statistical Computing, Vienna, Austria). Para a caracterização da amostra e identificação de grupos de vulnerabilidade, análises descritivas foram realizadas. A criação das redes de contatos e identificação dos seus principais elementos foram realizadas por meio da biblioteca igraph (Csardi \& Nepusz, 2006).

\section{Resultados e Discussão}

\subsection{Contato com os Discentes}

Ao longo de quatro meses, 52 discentes foram contatados por telefone. Entretanto, essa forma de contato não foi capaz de alcançar todos esses discentes, pois alguns números cadastrados junto à universidade estavam desatualizados ou porque, ao não reconhecerem a origem da ligação, não atendiam a chamada. A fim de superar tal obstáculo, outra estratégia adotada foi o envio de mensagens de texto pelo aplicativo WhatsApp ou por e-mail, nos casos em que os telefones de cadastro não estavam atualizados.

Dos 52 discentes denominados sementes ou indicados na amostragem por Bola de Neve, 32 aceitaram participar e responderam ao questionário.

\subsection{Perfil dos respondentes e constituição das redes}

A Tabela 1 apresenta as principais características dos participantes do estudo, baseadas em autodeclaração. A maioria dos estudantes estava matriculada no curso de Sistemas de Informação (53,1\%). A idade mediana foi de 24 anos (IQR $=4$ anos), sendo 65,6\% do gênero masculino, $53,1 \%$ de cor parda, preta ou indígena e $81,2 \%$ solteiros. A mediana do número de moradores na mesma casa foi igual a $3(\mathrm{IQR}=2)$. Cerca de $12 \%$ indicaram não se alimentar regularmente (pelo menos três refeições diárias). Em relação às bolsas e auxílios, 43,8\% e 87,5\% recebiam, respectivamente, auxílio alimentação e moradia, 25\% recebiam a Bolsa de Incentivo Acadêmico (BIA) e 37,5\% solicitaram o auxílio emergencial universitário disponibilizado pela UNIRIO, em agosto de 2020, aos estudantes com renda bruta familiar per capita de até 1,5 salário mínimo. 
Tabela 1: Perfil dos universitários participantes do estudo.

\begin{tabular}{|c|c|}
\hline Característica & $\mathbf{n}=\mathbf{3 2}$ \\
\hline \multicolumn{2}{|l|}{ Curso } \\
\hline Engenharia de Produção & $8(25,0 \%)$ \\
\hline Licenciatura em Matemática Presencial & $7(21,9 \%)$ \\
\hline Sistemas de Informação & $17(53,1 \%)$ \\
\hline Idade & $24(22-26)^{*}$ \\
\hline \multicolumn{2}{|l|}{ Gênero } \\
\hline Feminino & $11(34,4 \%)$ \\
\hline Masculino & $21(65,6 \%)$ \\
\hline \multicolumn{2}{|l|}{ Cor/Raça } \\
\hline Amarela & $1(3,1 \%)$ \\
\hline Branca & $9(43,8 \%)$ \\
\hline Indígena & $1(3,1 \%)$ \\
\hline Parda & $8(25,0 \%)$ \\
\hline Preta & $8(25,0 \%)$ \\
\hline \multicolumn{2}{|l|}{ Estado Civil } \\
\hline Casado/ Coabitação/ União Estável & $6(18,8 \%)$ \\
\hline Solteiro & $26(81,2 \%)$ \\
\hline Número de Pessoas na Casa & $3(2-4)^{*}$ \\
\hline \multicolumn{2}{|l|}{ Alimentação Regular } \\
\hline Não & $4(12,5 \%)$ \\
\hline Sim & $28(87,5 \%)$ \\
\hline \multicolumn{2}{|l|}{ Auxílio Emergencial Universitário } \\
\hline Não & $20(62,5 \%)$ \\
\hline Sim & $12(37,5 \%)$ \\
\hline \multicolumn{2}{|l|}{ Bolsas/Auxílios } \\
\hline Auxílio Alimentação & $14(43,8 \%)$ \\
\hline Auxílio Moradia & $28(87,5 \%)$ \\
\hline Bolsa de Incentivo Acadêmico (BIA) & $8(25,0 \%)$ \\
\hline
\end{tabular}

*Mediana (Q1 - Q3); Q1: primeiro quartil; Q3: terceiro quartil. Fonte: Autores.

A Figura 4 apresenta a distribuição das respostas segundo as possíveis causas de rompimento do vínculo com a universidade. Cerca de 34\% dos respondentes concordaram que o impacto econômico comprometeria a permanência no curso, enquanto metade dos participantes informou que as alterações na vida pessoal durante a pandemia da covid-19 poderiam impedir a continuação no curso. Ressalta-se que a falta de motivação acadêmica foi apontada por $41 \%$ dos respondentes como razão para impedir a continuação do curso. As condições psicológicas ou familiares foram apontadas por $38 \%$. 
Figura 4: Fatores que poderiam impedir a permanência no curso.

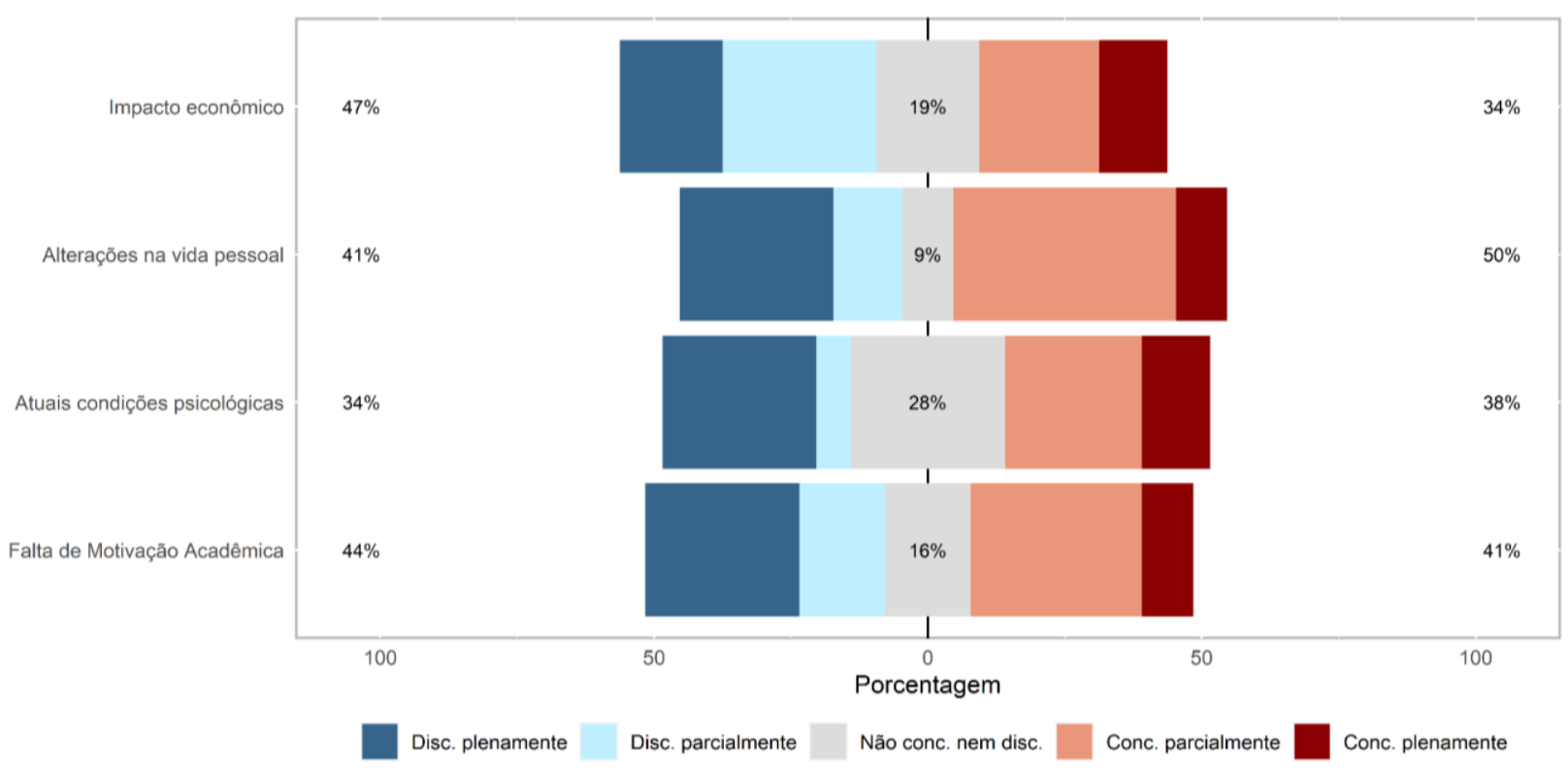

Fonte: Autores.

A Figura 5 apresenta as redes de contatos criadas a partir das respostas que indicavam discentes que poderiam estar em vulnerabilidade e com quem eles tinham mais proximidade. Cada bola colorida indica um indivíduo, sendo este codificado pelas cores referentes ao curso ou à posição na universidade (docente ou técnico administrativo). As setas indicam a relação entre os contatos, podendo ser de indicação de vulnerabilidade (seta vermelha) ou de proximidade (seta preta). Cada participante foi codificado por um número e suas iniciais. No total, seis redes foram formadas, com duas delas constituídas apenas pelas indicações de proximidade e as demais contabilizando 11 indicações de vulnerabilidade. Quatro redes menores foram formadas por discentes de Sistemas de Informação e Matemática.

As duas maiores redes de contato foram formadas da seguinte forma: (i) 24 discentes de Engenharia de Produção, dez docentes e um técnico administrativo, (ii) 26 discentes de Sistemas de Informação, 12 discentes de Matemática, 13 docentes e 3 técnicos administrativos. Na rede (i), o técnico 02T_JB foi o responsável pela união das redes de contatos menores, sendo indicado por proximidade por seis discentes, recebendo o maior número de indicações. O discente 09EP_JACS, também da mesma rede, foi o que mais fez indicações, 11 no total, apresentando o maior número de interações com qualquer outra pessoa. Em relação à rede (ii), o docente 05D_MC, com três indicações, foi o responsável por unir as redes menores formadas pelos discentes de Matemática, enquanto que o docente 06D_CM, com duas indicações, uniu essas redes às demais constituídas pelos alunos de Sistemas de Informação. O docente com mais indicações nesta rede foi o 01D_MD, com cinco indicações de discentes de Sistemas de Informação. Já o elo maior entre os alunos deste curso foi realizado pelo técnico 01T_DB, que apesar de receber apenas duas indicações, uniu duas redes de contato intermediárias. 
Figura 5: Redes de contatos criadas a partir das indicações de vulnerabilidade e proximidade.
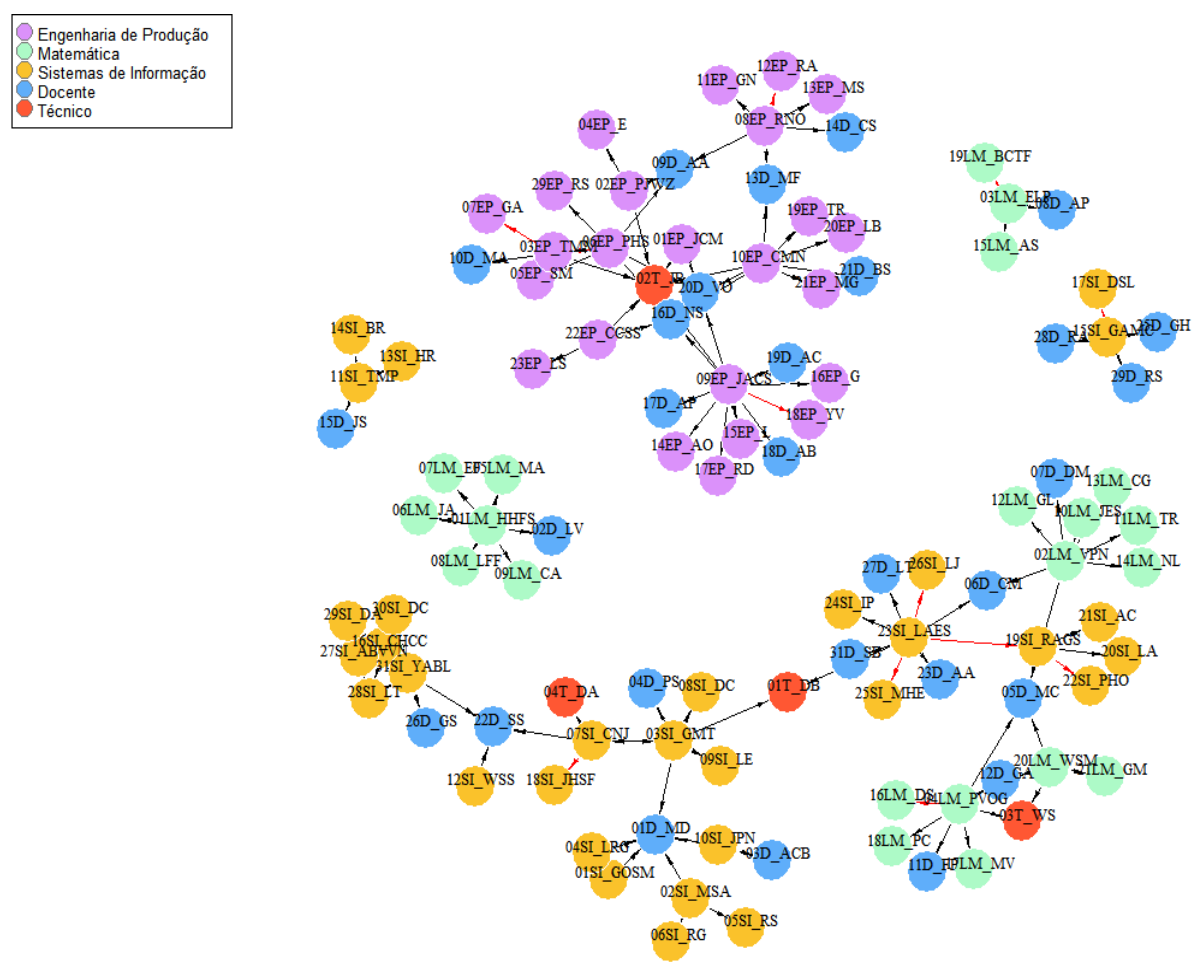

Fonte: Autores.

\section{Considerações Finais}

A pandemia da covid-19 gerou reflexões a respeito do quanto e como a realidade social e econômica afetaria a educação brasileira e sobre o que seria possível fazer para minimizar este efeito. Este projeto surgiu a partir da preocupação sobre os seus efeitos na população universitária mais vulnerável com vistas a entender e propor ações que minimizem os riscos de evasão. Este artigo apresentou os resultados gerados pela metodologia Bola de Neve aplicada à rede social do CCET da UNIRIO. Tais resultados apontaram que mais até do que os fatores econômicos, as modificações na vida pessoal e a falta de motivação acadêmica poderiam ser motivos para um possível abandono da Universidade. Ressalta-se que estas respostas são referentes ao mês de agosto de 2020, quando os fatores econômicos poderiam ainda não ser tão relevantes devido ao auxílio emergencial instituído e que foi responsável por diminuir o impacto da pandemia na economia brasileira (Sanches et al., 2021). Para buscar entender como atuar para aumentar a motivação acadêmica, as redes constituídas por discentes, docentes e técnicos foram utilizadas na definição dos convidados do primeiro evento remoto realizado com a metodologia World Café (Bussolotti et al., 2018). Este evento identificou uma expectativa dos discentes por mais diálogo em sala de aula e subsidiou as etapas seguintes do projeto que promoveu mais dois eventos remotos e cuja metodologia e resultados serão descritos em outro artigo.

$\mathrm{Na}$ avaliação desta primeira rodada, em caráter experimental, e realizada em apenas um centro acadêmico com cursos na área das ciências exatas, percebe-se, entre as suas limitações, as dificuldades relacionadas à representatividade de redes constituídas após cinco meses do início da pandemia e antes do início das aulas remotas. Por outro lado, considera-se que a metodologia apresentada, ao se enquadrar no que se denomina pesquisa-ação (Baldissera, 2001) ou pesquisa quanti-ação (Barbosa et al., 2019, 2016; Velasque et al., 2019), pode ser bem valiosa, o que permite conhecer o perfil dos discentes em risco de romperem seus vínculos acadêmicos e propor ações específicas voltadas para a sua realidade.

Para trabalhos futuros, espera-se ampliar a aplicação desta metodologia para os demais centros da Universidade, a fim de aumentar a rede de contatos entre discentes, professores e técnicos, assim como monitorar os discentes já identificados neste 
projeto inicial, em busca de evitar o rompimento de seus vínculos acadêmicos. Espera-se também retratar, em artigos futuros, os eventos remotos supracitados realizados entre discentes, docentes e técnicos administrativos.

\section{Referências}

Ahmed, F., Ahmed, N., Pissarides, C., \& Stiglitz, J. (2020). Why inequality could spread COVID-19. The Lancet Public Health, 5(5), e240.

Atkinson, R., \& Flint, J. (2001). Accessing hidden and hard-to-reach populations: Snowball research strategies. Social research update, 33(1), 1-4.

Baldissera, A. (2001). Pesquisa-ação: uma metodologia do “conhecer” e do "agir" coletivo. Sociedade Em Debate, 7(2), 5-25.

Barbosa, M. T. S., Byington, M. R. L., \& Struchiner, C. J. (2000). Modelos dinâmicos e redes sociais: revisão e reflexões a respeito de sua contribuição para o entendimento da epidemia do HIV. Cadernos de Saúde Pública, 16, S37-S51.

Barbosa, M. T. S., Ross, S. D., Silva, A. S. da, Velasque, L. de S., Simões, B. F. T., Cunha, M. B., \& Tuttman, M. T. (2016). A mudança de visão sobre a Lei de cotas em uma Universidade pública: evidências a partir de uma nova abordagem de pesquisa-quanti-ação. Anais do 22 Simpósio Nacional de Probabilidade e Estatística, 46.

Barbosa, M. T. S., Ross, S. D., Simões, B. F., Velasque, L. D. S., Silva, A. S., Assunção, M. B. M., \& Tuttman, M. (2019). Educação Estatística com formação cidadã em uma pesquisa quanti-ação no Ensino Superior. Revista de Ensino de Ciências e Matemática, 10(6), 114-125.

Bernard, H. R. (2005). Research Methods in Anthropology: Qualitative and Quantitative Approaches (4a ed.). AltaMira Press.

Borgatti, S. P., Everett, M. G., \& Johnson, J. C. (2018). Analyzing social networks. Sage.

Bussolotti, J. M., Souza, M. A., \& Cunha, V. M. P. (2018). O World Café como uma possibilidade interdisciplinar de aprendizagem ativa. CIET:EnPED.

Carvalho, L. (2020). Curto-circuito: o vírus e a volta do Estado. Todavia.

Csardi, G., \& Nepusz, T. (2006). The igraph software package for complex network research. InterJournal, Complex Systems, 1695(5), 1-9

Dempsey, L., Dowling, M., Larkin, P., \& Murphy, K. (2016). Sensitive Interviewing in Qualitative Research. Research in Nursing and Health, 39(6), 480490 .

Godoi, M. S. de, \& Santos, M. A. dos. (2021). Dez anos da lei federal das cotas universitárias: avaliação de seus efeitos e propostas para sua renovação e aperfeiçoamento. Revista de Informação Legislativa, 58(229), 11-35.

Goodman, L. A. (1961). Snowball Sampling. The Annals of Mathematical Statistics, 32(1), 148-170.

Griffiths, P., Gossop, M., Powis, B., \& Strang, J. (1993). Reaching hidden populations of drug users by privileged access interviewers: methodological and practical issues. Addiction, 88(12), 1617-1626.

Hindhede, A. L., \& Aagaard-Hansen, J. (2017). Using Social Network Analysis as a Method to Assess and Strengthen Participation in Health Promotion Programs in Vulnerable Areas. Health Promotion Practice, 18(2), 175-183.

Miranda, P., Koeller, P., Zucoloto, G., Machado, W., \& De Nigri, F. (2020). Nota Técnica - 2020 - Julho - Número 72 - Diset - Aspectos socioeconômicos da COVID-19: o que dizem os dados do Município do Rio de Janeiro? Instituto de Pesquisa Econômica Aplicada. https://www.ipea.gov.br/portal/index.php?option=com_content\&view=article\&id=36249

Mussliner, B. O., De Sousa, M., Mussliner, S., Benito, E., \& Luján Rodríguez, G. (2021). O problema da evasão universitária: um desafio à democratização do ensino superior público. Brazilian Journal of Development, 7(4), 42738-42758.

Sanches, M., Cardomingo, M., \& Carvalho, L. (2021). Quão mais fundo poderia ter sido esse poço? Analisando o efeito estabilizador do Auxílio Emergencial em 2020 (Nota de Política Econômica no 007). São Paulo: MADE/USP. https://madeusp.com.br/publicacoes/artigos/quao-mais-fundo-poderia-ter-sido-essepoco-analisando-o-efeito-estabilizador-do-auxilio-emergencial-em-2020/

Scott, J. (1988). Social network analysis. Sociology, 22(1), 109-127.

Velasque, L. S., Silva, A. S., \& Barbosa, M. T. S. (2019). A pesquisa quanti-ação como ferramenta para o ensino de estatística na Educação Básica. Revemat: Revista Eletrônica de Educação Matemática, 14(0), 1-16. 